\title{
Connecting Turbulence and Meandering Parameterization to Describe Passive Scalars Dispersion in Low Wind Speed Conditions
}

\author{
V. S. Moreira, ${ }^{1}$ G. Degrazia, ${ }^{2}$ A. U. Timm, ${ }^{2}$ D. R. Roberti, ${ }^{2}$ and S. Maldaner ${ }^{2}$ \\ ${ }^{1}$ Departamento de Engenharia, Universidade Federal do Pampa (UNIPAMPA), 97650-000 Itaqui, RS, Brazil \\ ${ }^{2}$ Departamento de Física, Universidade Federal de Santa Maria, 97110-900 Santa Maria, RS, Brazil \\ Correspondence should be addressed to V. S. Moreira; virneimoreira@gmail.com
}

Received 14 September 2012; Accepted 2 October 2012

Academic Editors: C. Mangia and B. Rappenglueck

Copyright (C) 2013 V. S. Moreira et al. This is an open access article distributed under the Creative Commons Attribution License, which permits unrestricted use, distribution, and reproduction in any medium, provided the original work is properly cited.

\begin{abstract}
The following study deals with meandering of the horizontal mean wind. The main motivation for such investigation came from the difficulty in describing contaminant dispersion in meandering conditions. Observational field measurements point out that the autocorrelation function of the horizontal wind components, obtained for the meandering cases, displays an oscillating behavior with the presence of large negative lobes. Such negative lobes are described by an equation containing functions that represent patterns of movement associated to meandering and turbulence. As a consequence, this mathematical formulation connects the turbulence and meandering phenomenon establishing the employment of hybrid parameters in models that describe the meandering dispersion. Therefore, considering this dualistic aspect between meandering and turbulence manifestations, a new set of relations for the turbulence parameterization joined with the meandering of the wind have been developed and are available. This new turbulence parameterization for a stable shear forcing planetary boundary layer, united with a meandering mean time scale is able to describe contaminant meandering enhanced spread in a low wind speed stable planetary boundary layer.
\end{abstract}

\section{Introduction}

Low-frequency meandering of the horizontal mean wind vector occurring in low wind speed conditions is a complex physical phenomenon associated with turbulence in the stable planetary boundary layer (PBL) [1]. Using a number of simplifying assumptions in the three-dimensional NavierStokes equations [2], the influence of the Reynolds-stress terms on the meandering phenomenon has been analyzed. The study shows that when the turbulent forcing can be neglected, the Navier-Stokes equations provide an asymptotic meandering solution that describes a nondecaying horizontal wind oscillation. Differently, for increasing values of the turbulent forcing, the presence of the horizontal Reynolds-stress terms demonstrates that the action of turbulence changes the geometry of the flow field. In this aspect, the turbulent forcing imposes a finite relaxation time and transforms a two-dimensional flow to one-dimensional. Therefore, this new order of the flow, generated by the turbulence leads to a different spatial symmetry, establishing a precise mean wind direction and ceasing the meandering behavior to exist. Because PBL similarity theory fails to represent transport processes when winds are calm, it remains a difficult physical task to derive air pollution dispersion models that simulate meandering enhanced diffusion of passive scalars in a low wind stable PBL [3]. In such situations, the airborne contaminants are dispersed over rather wide angular sectors and therefore it is no longer possible to establish a definite mean wind direction since low-frequency horizontal wind oscillations start to dominate and diffusion of contaminants in the PBL becomes controlled by these degrees of freedom, characterized by low frequencies (large characteristic time associated with the meandering period) [4]. As a consequence, the occurrence of low wind is generally considered 
the most critical situation associated with the air pollution dispersion problem and therefore considerable research has been carried out over the recent past on developing better modeling methods for dispersion in low wind speed conditions [3,5-10]. The presence of large negative lobes in an observed meandering autocorrelation function can be very well fitted by the following formula $[4,11]$ :

$$
\rho_{L_{i}}(\tau)=e^{-p \tau} \cos (q \tau)
$$

where

$$
\begin{aligned}
& p=\frac{1}{\left(m^{2}+1\right) T_{L_{u, v}}}, \\
& q=\frac{m}{\left(m^{2}+1\right) T_{L_{u, v}}},
\end{aligned}
$$

with $m$ being the loop parameter which controls the meandering oscillation frequency associated to the horizontal wind and $T_{L_{u, v}}$ is the local velocity decorrelation time scale for a fully developed turbulence. Equation (1) has been proposed by $[12,13]$. Furthermore, considering idealized mathematical solutions it can be derived from Navier-Stokes equations (forecast equation for mean wind). Analyzing the Frenkiel function it is possible to notice that it is expressed as the product of the classical exponential function (representing the autocorrelation function for a fully developed turbulence) by the cosine function (describing the horizontal wind oscillations associated to the meandering phenomenon). As a consequence, the Frenkiel autocorrelation function captures well the physical properties of a fully developed turbulence as well as hybrid flow cases, in which turbulence and meandering occurrences coexist. The pattern of pure turbulence or those associated to predominant meandering effects is determined by the magnitudes of the local velocity decorrelation time scale $T_{L_{u, v}}$ and of the loop parameter $m$. Indeed, the Frenkiel formulation presents a complementary character and as a consequence provides a flexibility that allows representing observations in the PBL $[4,14,15]$. This imparts to (1) a wide-ranging heuristic validity.

Identifying $q=2 \pi / T_{M}$ as a meandering frequency, in which $T_{M}$ is a meandering period, it is possible from (3) to obtain the following formula for the loop parameter $m$ :

$$
m=\frac{T_{M}+\sqrt{T_{M}^{2}-16 \pi^{2} T_{L_{u, v}}^{2}}}{4 \pi T_{L_{u, v}}} .
$$

A large number of time series of sonic anemometer wind speeds were analyzed by [4]. These observational data suggest that the mean magnitude of the meandering period is of the order of $T_{M} \approx 2000 \mathrm{~s}$. Considering that the values of $T_{L_{u, v}}$ for a stable PBL are a hundred-times lower than $T_{M}$ (4) can be rewritten as

$$
m \approx \frac{T_{M}}{2 \pi T_{L_{u, v}}},
$$

defining $m$ as the ratio of meandering period to the local velocity decorrelation time scale for a fully developed turbulence. Therefore, (4) and (5) show that the presence of large turbulent coherent structures characterized by large values of $T_{L_{u, v}}$ inhibiting the increasing of $m$ and as a consequence the reinforced meandering dispersion tends to fade away in the stable PBL. Anfossi et al. [11] proposed a Lagrangian stochastic dispersion model composed of two coupled Langevin equations to describe the pollutants dispersion in meandering situations. Such approach is described by the following system of equations:

$$
\begin{gathered}
d u=[-p(u-\bar{u})-q(v-\bar{v})] d t+\sqrt{2 p d t} \sigma_{u} \xi_{u}, \\
d v=[q(u-\bar{u})-p(v-\bar{v})] d t+\sqrt{2 p d t} \sigma_{v} \xi_{v}
\end{gathered}
$$

where $u$ and $v$ are the horizontal components of the wind velocity fluctuations, $\xi_{u}$ and $\xi_{v}$ are random Gaussian variables having zero mean and unit variances, and $\sigma_{u}$ and $\sigma_{v}$ are standard deviations of the horizontal wind components. It is important to note that (6) are valid assuming horizontal homogeneous conditions [11]. For the vertical component of the velocity fluctuation $w$ the Langevin equation has been solved according to the usual LAMBDA model $[11,16]$

$$
d w=a_{i}(z, w)+b_{0}(z) d w_{j},
$$

where $d w_{j}$ is the incremental Gaussian Wiener process (with zero mean and variance $d t), b_{0}(z)=\sqrt{2 \sigma_{w}^{2} / T_{L_{w}}}\left(\sigma_{w}^{2}\right.$ is the vertical turbulent velocity variance and $T_{L_{w}}$ is the vertical local Lagrangian time scale) and $a_{i}(z, w)$ is computed by solving the Fokker-Planck equation associated with (6) using a probability distribution function of Gram-Charlier type truncated to the third order [17]. The position of each particle, at each time step is obtained by the numerical integration of (6) and (7) and the following equation:

$$
d x_{i}=u_{i} d t
$$

where $i=1,2,3, x_{i}$ is the position vector of each particle and $u_{i}$ is its corresponding Lagrangian velocity vector. Thus, to describe the dispersion of passive scalars in the PBL the Langevin equation is integrated according to the rules of the Ito calculus [18], which was developed to obtain solutions of the stochastic differential equations.

The aim of this study is to derive a turbulence parameterization that can be employed in Lagrangian stochastic dispersion models to simulate the dispersion of contaminants in a low wind speed stable PBL presenting meandering phenomenon. Specifically, formulations for the turbulent velocity standard deviations and an expression providing the eddy diffusivity for the momentum flux have been employed to derive relations for the local turbulent time scales in a shear-driven stable PBL. Therefore, the proposed parameterization to describe the meandering dispersion of contaminants employs an observational mean value for the meandering period, turbulent velocity variances, and local velocity decorrelation time scales. An additional purpose is to introduce this parameterization derived from eddy diffusivity in the Lagrangian stochastic dispersion model described by (6), (7), and (8) to simulate the dispersion of contaminants in low wind speed meandering conditions in the stable PBL. 


\section{Derivation of Local Velocity Decorrelation Time Scale from Momentum Eddy Diffusivity and Velocity Variances}

For a shear-dominated stable PBL, the eddy diffusivity has been considered for the momentum flux $\left(K_{m}\right)$ and the velocity variances $\left(\sigma_{u}^{2}, \sigma_{v}^{2}, \sigma_{w}^{2}\right)$ given by [19]

$$
\begin{gathered}
\frac{\sigma_{u}^{2}+\sigma_{v}^{2}}{u_{*}^{2}}=9.3, \\
\frac{\sigma_{w}^{2}}{u_{*}^{2}}=2.5, \\
\frac{K_{m}}{u_{* 0} h}=\frac{k(z / h(1-z / h))}{1+4.7(h / L)(z / h)},
\end{gathered}
$$

where $u_{*}$ is the local friction velocity, $u_{* 0}$ is the surface friction velocity, $z$ is the height above the surface, and $h$ is the height of the turbulent stable PBL. Equation (11) describes a $K_{m}$ profile in lower and central regions of the stable PBL presenting the same pattern as those derived by the authors of [20] which is based on Tsallis's nonextensive statistical mechanics [21].

Equation (9) provides the value of the horizontal velocity variance obtained by adding together the lateral and longitudinal velocity variances. However, the following formulation for $\sigma_{u}^{2}$ is given by

$$
\sigma_{u}^{2}=\frac{2.33 c_{u} \phi_{\varepsilon}^{2 / 3} u_{*}^{2}}{\left(f_{m}\right)_{u}^{2 / 3}}
$$

where $c_{u}=0.27, \phi_{\varepsilon}=1.25$ and $\left(f_{m}\right)_{u}=0.045$ allows to determine the individual constants for $\sigma_{u}^{2}$ and $\sigma_{v}^{2}$ from (9) and (12). Therefore, the present analysis yields

$$
\begin{aligned}
\sigma_{u}^{2} & =6.0\left(1-\frac{z}{h}\right)^{2} u_{* 0}, \sigma_{v}^{2} \\
& =3.3\left(1-\frac{z}{h}\right)^{2} u_{* 0},
\end{aligned}
$$

where $u_{*}^{2}=(1-z / h)^{2} u_{* 0}^{2}$ [19]. Considering that the local velocity decorrelation time scales are obtained from the ratio between eddy diffusivities and velocity variances (10), (11), and (13) provide the following relations for the turbulent local time scales in a shear-dominated stable PBL:

$$
\begin{aligned}
T_{L u} & =\frac{z k}{6.0[1+4.7(z / L)](1-(z / h)) u_{* 0}}, \\
T_{L v} & =\frac{z k}{3.3[1+4.7(z / L)](1-(z / h)) u_{* 0}}, \\
T_{L w} & =\frac{z k}{2.5[1+4.7(z / L)](1-(z / h)) u_{* 0}} .
\end{aligned}
$$

Therefore, employing (5), with $T_{M} \approx 2000 \mathrm{~s}, \sigma_{u}^{2}$ and $\sigma_{v}^{2}$ given by (13), and $T_{L u, v}$ given by (14), the low frequency horizontal wind oscillation effects can be parameterized and introduced into (6) to simulate the observed dispersion of passive scalars caused by the meandering transport in the shear-driven stable PBL.

\section{Meandering Dispersion Simulation}

The results of the present model are evaluated with the concentration data obtained under stable conditions in low wind speeds over flat terrain at the Idaho Engineering Laboratory (INEL). These observed concentrations have been published in a U.S. National Oceanic and Atmospheric Administration (NOAA) report [22]. Because of wind direction large variability occurring with the meandering phenomenon, full $360^{\circ}$ were employed. Arcs were laid out at radii of 100, 200, and $400 \mathrm{~m}$ from the emission point source. Samplers were placed at intervals of $6^{\circ}$ for a total of 180 sampling locations. The receptor height was $0.76 \mathrm{~m}$. The tracer $S F_{6}$ was released at a height of $1.5 \mathrm{~m}$. The $1 \mathrm{~h}$ average concentrations were determined by means of an electron capture gas chromatography. Wind speeds measured at levels $2,4,8,16,32$, and $61 \mathrm{~m}$ were utilized to calculate the coefficient for the exponential wind vertical profile. According to $[5,7]$ the roughness length used was $z_{0}=0.005 \mathrm{~m}$. The Monin-Obukhov length $L$ (height at which shear and buoyant production rates of turbulent kinetic energy are equal) and the friction velocity $u_{*}$ were not available for the INEL experiment but can be roughly estimated by different formulations. Therefore, $L$ may be estimated from an empirical formulation suggested by [23] and the stable turbulent PBL height $h$ was determined according to the expression derived by the authors of [24]. Table 1 shows the micrometeorological data employed for the validation of the present approach.

INEL observed concentrations, $\chi_{m}\left(\mathrm{~m}^{-2}\right)$, were normalized according to the following relation [22]:

$$
\chi_{m}=C_{m} \frac{U_{4}}{Q}
$$

where $C_{m}$ is the dimensional concentration expressed in $\mathrm{gm}^{-3}, U_{4}$ is the mean wind speed at $4 \mathrm{~m}$, and $Q$ is the tracer emission rate $\mathrm{g} \mathrm{s}^{-1}$. Consequently, predicted concentrations are for the INEL experiments expressed in $\mathrm{m}^{-2}$.

During the simulations the turbulent flow field is considered as not homogeneous in the vertical direction and the transport is caused by the longitudinal component of the mean wind velocity. In these specific simulations, the horizontal domain of model was determined according to sampler distances and the vertical domain was set equal to the observed PBL height $h$. The emission point source was localized at the domain centre. The time step was maintained constant and equal to $\Delta t=0.5 \mathrm{~s}$. The magnitude of this time step is of the order of the time scales of Kolmogorov's turbulent energy spectrum inertial subrange. Furthermore, this value of $\Delta t$ obeys the following inequality $\Delta t \ll T_{L}$. This condition assures that the turbulent velocities can be considered a Markov process [25]. For each numerical run, the number of particles released was $10^{6}$. For the INEL experiments, the cells of concentration at ground-level have a vertical dimension of 
TABLE 1: Micrometeorological data observed during the INEL experiment.

\begin{tabular}{|c|c|c|c|c|c|c|c|c|c|}
\hline Run & $u_{*}$ & $L(\mathrm{~m})$ & $H(\mathrm{~m})$ & $U(2 \mathrm{~m})$ & $U(4 \mathrm{~m})$ & $U(8 \mathrm{~m})$ & $U(16 \mathrm{~m})$ & $U(32 \mathrm{~m})$ & $U(61)$ \\
\hline 4 & 0.047 & 2.40 & 13.40 & 0.7 & 1.2 & 999 & 1.5 & 0.9 & 2.1 \\
\hline 5 & 0.053 & 3.14 & 16.38 & 0.8 & 0.9 & 1.2 & 2.2 & 3.0 & 2.1 \\
\hline 7 & 0.040 & 1.77 & 10.64 & 0.6 & 0.9 & 0.4 & 0.5 & 0.9 & 2.4 \\
\hline 8 & 0.033 & 1.22 & 8.09 & 0.5 & 0.8 & 0.6 & 1.2 & 1.6 & 2.7 \\
\hline 9 & 0.033 & 1.22 & 8.09 & 0.5 & 0.8 & 0.9 & 1.6 & 2.2 & 2.7 \\
\hline 10 & 0.073 & 5.93 & 26.40 & 1.1 & 1.7 & 2.1 & 3.2 & 4.7 & 3.1 \\
\hline 11 & 0.093 & 9.60 & 37.91 & 1.4 & 1.9 & 2.3 & 2.9 & 999 & 3.6 \\
\hline 12 & 0.047 & 2.40 & 13.40 & 0.7 & 1.1 & 1.1 & 1.6 & 1.6 & 1.9 \\
\hline 13 & 0.067 & 4.90 & 22.88 & 1.0 & 1.6 & 2.0 & 3.0 & 4.0 & 6.0 \\
\hline 14 & 0.067 & 4.90 & 22.88 & 1.0 & 1.6 & 2.0 & 3.5 & 5.1 & 7.1 \\
\hline
\end{tabular}

TABLE 2: Statistical indices evaluating the model performance.

\begin{tabular}{lccccc}
\hline & NMSE & $R$ & FA2 & FB & FS \\
\hline $\begin{array}{l}\text { Meandering parameterization } \\
\text { obtained in this study }\end{array}$ & 0.20 & 0.94 & 0.77 & 0.30 & 0.17 \\
Sagendorf and Dickson [22] & 0.60 & 0.42 & 0.80 & 0.06 & - \\
Sharan and Yadav [7] & 0.53 & 0.55 & 0.60 & -0.02 & - \\
Oettl et al. [3] & 0.21 & 0.86 & 0.87 & -0.13 & - \\
\hline
\end{tabular}

$\Delta z=3 \mathrm{~m}[11]$. On the other hand, the horizontal dimensions were calculated from the following relation:

$$
\Delta x=\Delta y=\frac{2 \pi r}{N_{\mathrm{ang}}},
$$

where $r$ is the arc radius and $N_{\text {ang }}=60$ is the number of samplers per arc. This way of computing the cell size covers all the compass at the three radii without significant overlapping. These criteria result $\Delta x=10.47 \mathrm{~m}, \Delta y=20.93 \mathrm{~m}$, and $\Delta z=41.87 \mathrm{~m}$ for the three arcs, respectively. The simulated concentrations were obtained by counting the number of particles in volumes generated from the vertical and horizontal dimensions above presented.

\section{Comparison between Simulated and Observed Concentrations}

The results of the simulation obtained from (6) using the meandering phenomenon description, as given by (5), (13), and (14), are shown in Figures $1-3$ and in Table 2. Figure 1 shows the simulation of the meandering experiment 8 . This particular experiment exhibits a significant horizontal dispersion that occurs over a wide angular range (one providing the widest horizontal contaminant spread), yielding that the contaminant is observed in all angles. Therefore, employing the meandering and turbulence parameters derived from the momentum eddy diffusivity (11) and the velocity variances (13) it is possible to reproduce the meandering spread over all the $360^{\circ}$. From the environmental point of view it is very important to determine the locus in which the maximum concentrations are observed. It can be seen in Figure 1 that the model simulates adequately the maximum concentrations for experiment 8 at distances of 100, 200, and $400 \mathrm{~m}$. On the other hand, Figure 2 shows that the stochastic dispersion model, utilizing the present meandering parameterization, reproduces with a certain precision the observed maximum concentrations for experiment 6 at 100, 200, and $400 \mathrm{~m}$.

In order to obtain a general assessment on the model describing the meandering enhanced dispersion, statistical indices have been evaluated at each arc, for the ten INEL runs: concmax, top5, and $S_{y}$. Concmax $\left(\mathrm{m}^{-2}\right)$ is the maximum ground-level concentration; top $5\left(\mathrm{~m}^{-2}\right)$ refers to the mean of the 5 highest measured and computed ground-level concentration and $S_{y}=\sqrt{\sum_{i=1}^{N} \chi_{i}\left(\vartheta_{i}-\bar{\vartheta}\right)^{2} / \sum_{i=1}^{N} \chi_{i}}$ (degrees), where $\vartheta_{i}$ are the sampler angles and $\bar{\vartheta}$ their average value (weighted with the concentrations). Figure 3 shows the results of these particular statistical indices (concmax, top5, and $S_{y}$ ) that are calculated from (6) using the meandering description proposed in this study.

Specifically, Figure 3 displays the scatterplot between observed and predicted concmax, top5, and values. Analyzing these scatter diagrams it is possible to notice that there is a reasonable difference between the observed and the predicted quantities; however, this incomplete description provided by the present approach is not significant to preclude an acceptable alignment in relation to the straight of optimal fitting. Table 2 presents the results of the statistical indices obtained with observed and predicted magnitudes of peak concentration. Furthermore, this table presents a comparison between the meandering parameterization presented in this investigation (5), (13), (14), and (15) and other three models that simulate also the meandering dispersion phenomenon $[3,7,22]$. This set of statistical indices is proposed by [26] to evaluate new dispersion models. They are defined by the following relations:

$$
\begin{gathered}
\text { NMSE }=\frac{\overline{\left(C_{o}-C_{p}\right)^{2}}}{\overline{C_{o} C_{p}}} \quad(\text { normalized mean square error }), \\
\mathrm{FB}=\frac{\overline{C_{o}}-\overline{C_{p}}}{0.5\left(\overline{C_{o}}+\overline{C_{p}}\right)} \quad \text { (fractional bias) },
\end{gathered}
$$



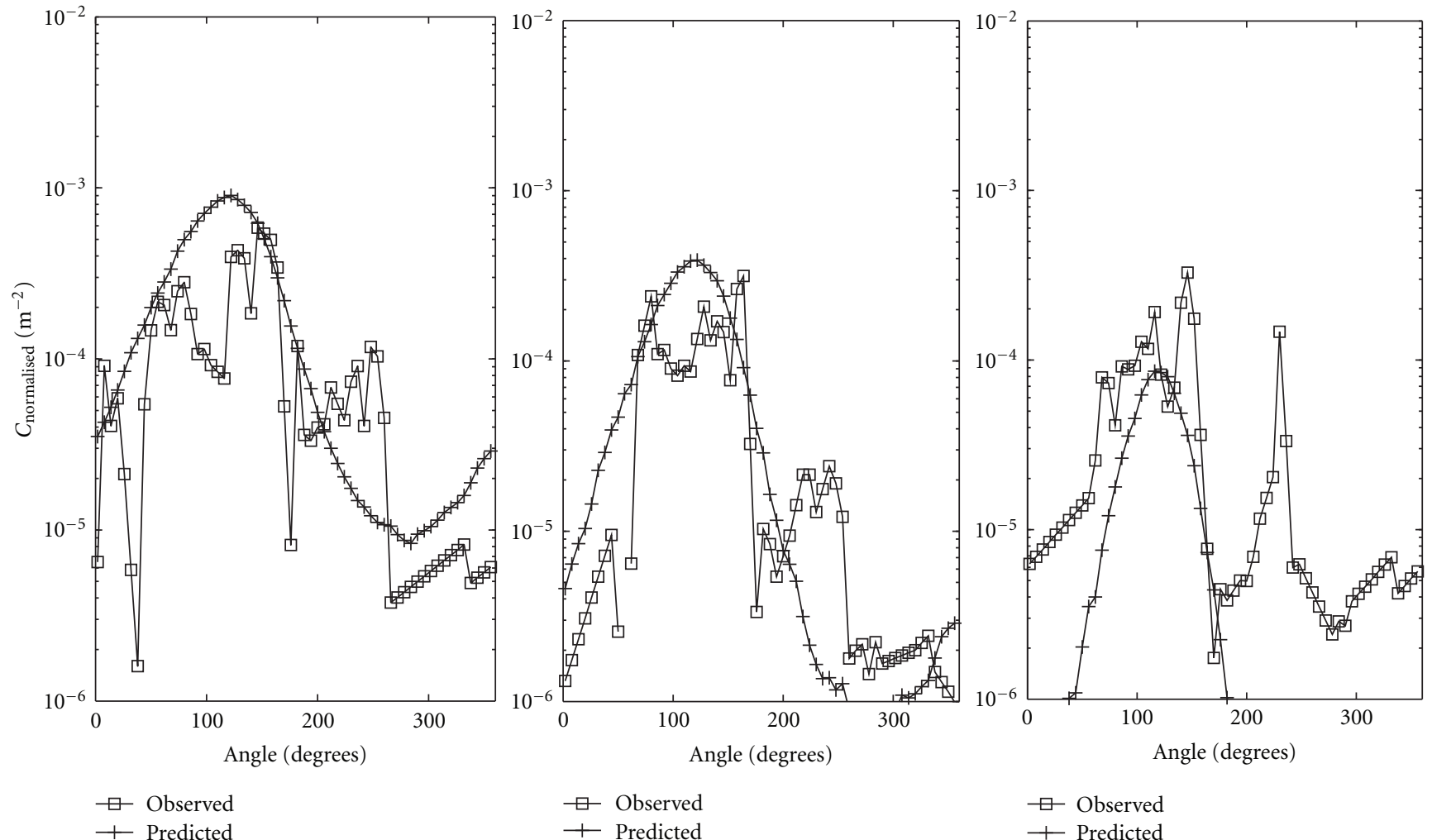

FIGURE 1: Figures 1-3: normalized ground level concentration $\left(C_{\text {normalized }}=\chi_{\mathrm{m}}, \mathrm{m}^{-2}\right)$ for experiment 8 at $100 \mathrm{~m}, 200 \mathrm{~m}$, and $400 \mathrm{~m}$ as a function of the sampler angles. Open squares indicate observed concentrations and crosses indicate simulated concentrations.

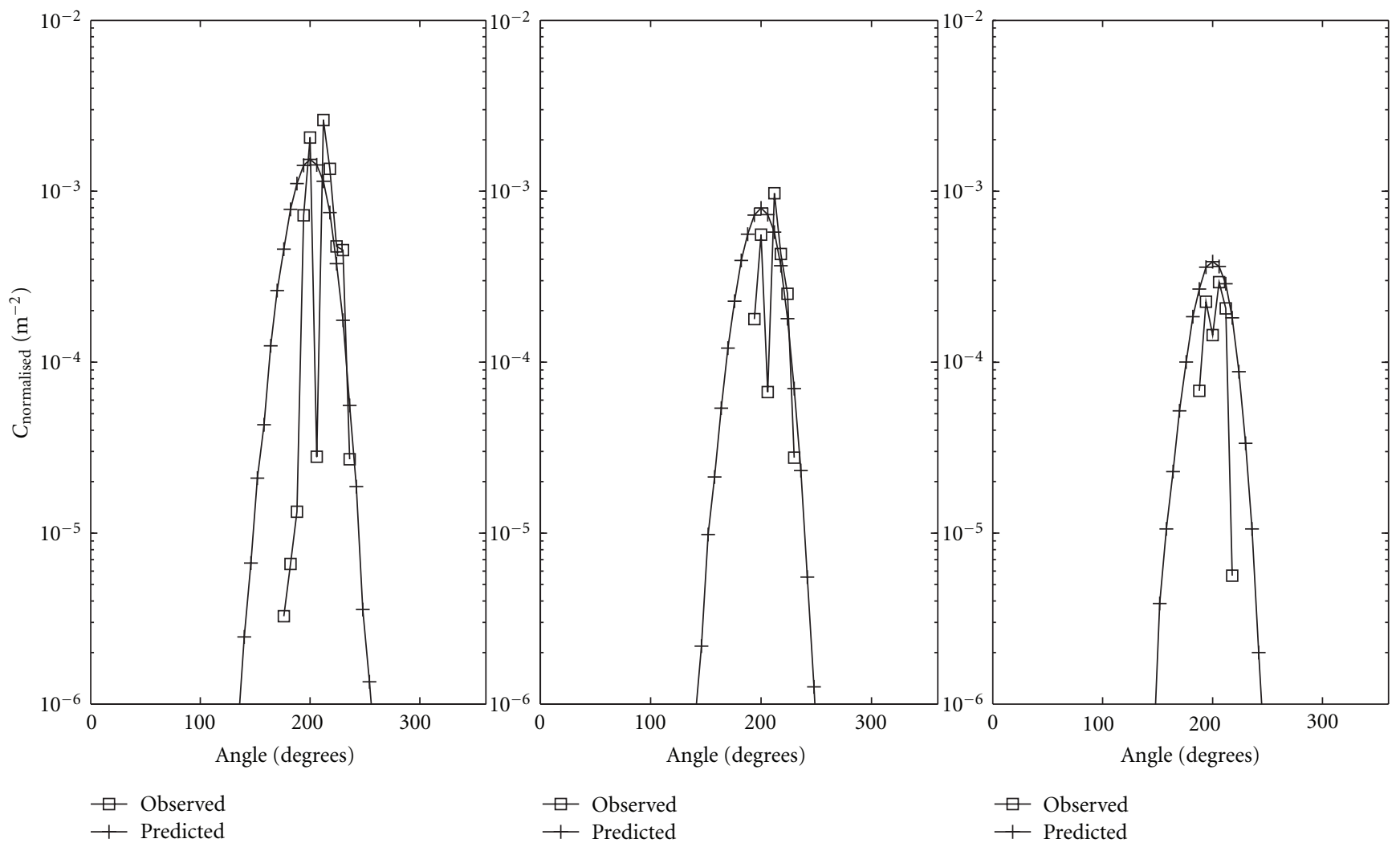

FIGURE 2: As Figure 1 but for experiment 6. 


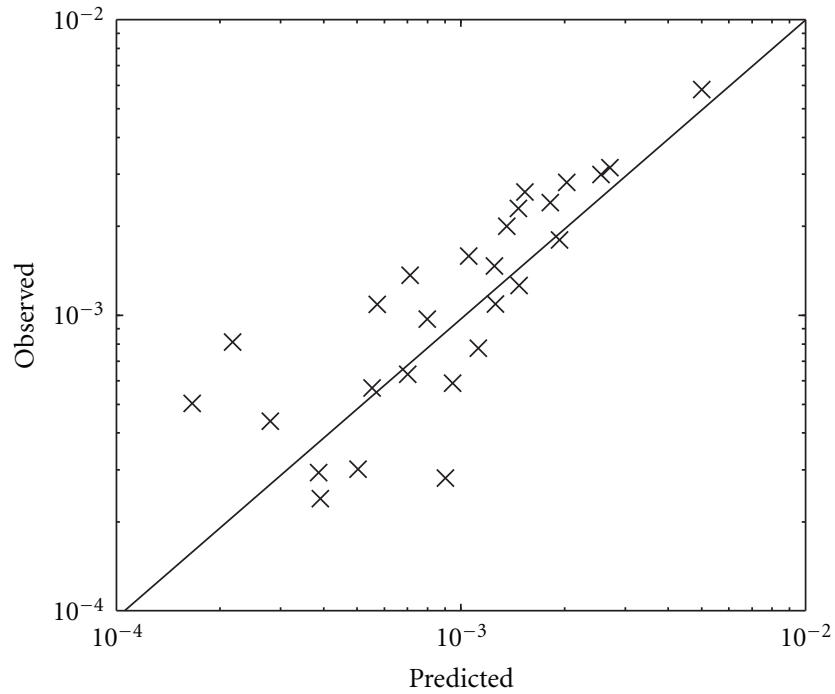

(a)

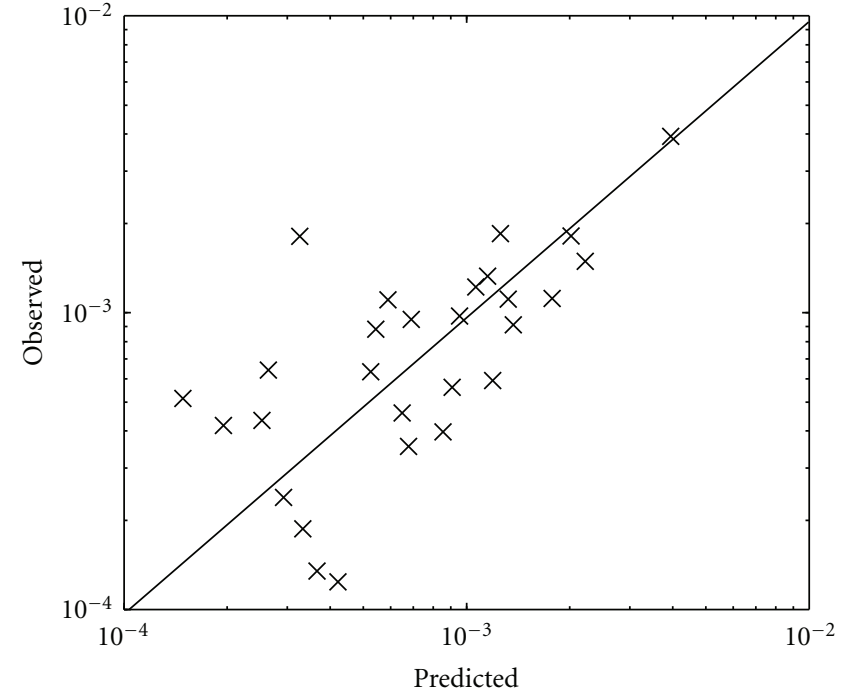

(b)

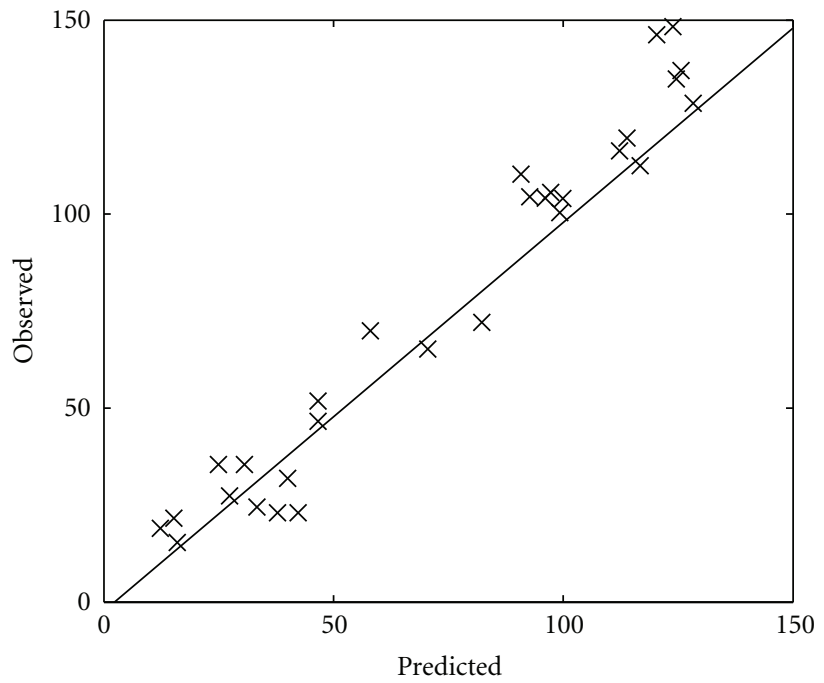

(c)

Figure 3: Plot of (a) concmax $\left(\mathrm{m}^{-2}\right)$, (b) top5 $\left(\mathrm{m}^{-2}\right)$, and (c) $S_{y}$ (degrees) calculated with present model. $X$-axis shows predicted values whereas observed values are on the $Y$-axis.

$$
\begin{gathered}
\mathrm{FS}=\frac{2\left(\sigma_{o}-\sigma_{p}\right)}{\sigma_{o}+\sigma_{p}} \quad \text { (fractional standard deviation), } \\
R=\frac{\overline{\left(C_{o}-\overline{C_{o}}\right)\left(C_{p}-\overline{C_{p}}\right)}}{\sigma_{o} \sigma_{p}} \quad \text { (Correlation Coeficient) }, \\
\mathrm{FA} 2=0.5 \leq \frac{C_{o}}{C_{p}} \leq 2 \quad(\text { Factor } 2),
\end{gathered}
$$

where $C$ is the analyzed quantity (concentration) and the subscripts " $o$ " and " $p$ " represent the observed and the predicted values, respectively. The overbars in statistical indices indicate averages. The statistical index FB indicates if the predicted quantity underestimates or overestimates the observed one. The statistical index NMSE represents the quadratic error of the predicted quantity in relation to the observed one. The statistical index FS indicates the measure of the comparison between predicted and observed plume spreading. The statistical index FA2 provides the fraction of data for which $0.5 \leq C_{o} / C_{p} \leq 2$. As nearest zero are NMSE, FB and FS and as nearest one are the R and FA2, better are the results. From this statistical analysis we may promptly conclude that the present model, representing the particular phenomenon of the meandering dispersion, in 
general simulates fairly well the concentration experimental data in a low wind meandering condition occurring in a shear driven stable PBL.

\section{Conclusions}

Meandering of the horizontal mean wind is a complex physical phenomenon of fundamental importance in air pollution dispersion modeling. Experimental evidences display the fact that the existence of large negative lobes in measured meandering autocorrelation functions can be well represented by an expression describing both turbulence and meandering degrees of freedom. As a consequence, meandering phenomenon, mainly characterized by low oscillation of the horizontal wind, can be seen as a special atmospheric movement connected directly with turbulence. Thus, movement patterns in the PBL can be characterized by hybrid states, in which turbulence and meandering with distinct weights of participation in the phenomenology can be present. For instance, the simplified form as given by (5), containing the meandering period, establishes this connection and provides a fundamental parameter to be used in the relevant Equation (6) that models the meandering dispersion. Therefore, in this study, considering this complementary characteristic between meandering and turbulence states, a new formulation for the turbulence parameterization associated with the meandering phenomenon has been derived and tested. This description for the turbulence in a shear driven stable PBL employs velocity variances (10) and (13) obtained from experimental fittings and turbulent local velocity decorrelation time scales (14) and (15) derived from theses variances and of a formulation for the momentum eddy diffusivity (11) based on Tsallis's nonextensive statistical mechanics [21]. Furthermore, the meandering parameterization is described by an observed representative mean value of the meandering period of the order of $T_{M} \approx 2000 \mathrm{~s}$. The parameterization developed in this investigation has been applied in situations in which turbulence and meandering phenomenon coexist, being used to simulate concentration data. Additionally, these simulated data were evaluated and tested through the comparison with the observational concentration data and with those data simulated by different meandering dispersion models. Particularly, the results obtained by Lagrangian stochastic dispersion model (6), (7), and (8) employing the new formulas for the turbulence parameterization show that the model correctly represents the meandering dispersion process in a low wind speed stable condition.

The present study, from a point of view of description of the meandering movement, shows that a physically reasonable turbulence parameterization for a stable shear driven PBL allied with a characteristic mean value of the meandering period is enough to represent the meandering dispersion.

\section{Acknowledgments}

The authors acknowledge the financial support provided by $\mathrm{CNPq}$ (Conselho Nacional de Desenvolvimento Científico e tecnológico) and CAPES (Coordenacão de Aperfeiçoamento de Pessoal de Nível Superior).

\section{References}

[1] L. Mahrt, "Intermittency of atmospheric turbulence," Journal of the Atmospheric Sciences, vol. 46, no. 1, pp. 79-95, 1989.

[2] A. G. O. Goulart, G. A. Degrazia, O. C. Acevedo, and D. Anfossi, "Theoretical considerations of meandering winds in simplified conditions," Boundary-Layer Meteorology, vol. 125, no. 2, pp. 279-287, 2007.

[3] D. Oettl, R. A. Almbauer, and P. J. Sturm, "A new method to estimate diffusion in stable, low-wind conditions," Journal of Applied Meteorology, vol. 40, no. 2, pp. 259-268, 2001.

[4] D. Anfossi, D. Oettl, G. Degrazia, and A. Goulart, "An analysis of sonic anemometer observations in low wind speed conditions," Boundary-Layer Meteorology, vol. 114, no. 1, pp. 179-203, 2005.

[5] G. Brusasca, G. Tinarelli, and D. Anfossi, "Particle model simulation of diffusion in low wind speed stable conditions," Atmospheric Environment Part A, vol. 26, no. 4, pp. 707-723, 1992.

[6] M. C. Cirillo and A. A. Poli, "An intercomparison of semiempirical diffusion models under low wind speed, stable conditions," Atmospheric Environment Part A, vol. 26, no. 5, pp. 765-774, 1992.

[7] M. Sharan and A. K. Yadav, "Simulation of diffusion experiments under light wind, stable conditions by a variable $\mathrm{K}$ theory model," Atmospheric Environment, vol. 32, no. 20, pp. 3481-3492, 1998.

[8] J. C. Carvalho, M. T. M. B. De Vilhena, and D. M. Moreira, "An alternative numerical approach to solve the langevin equation applied to air pollution dispersion," Water, Air, and Soil Pollution, vol. 163, no. 1-4, pp. 103-118, 2005.

[9] A. K. Luhar, "Analytical puff modelling of light-wind dispersion in stable and unstable conditions," Atmospheric Environment, vol. 45, no. 2, pp. 357-368, 2011.

[10] A. K. Luhar and P. J. Hurley, "Application of a coupled prognostic model to turbulence and dispersion in light-wind stable conditions,with an analytical correction to vertically resolve concentrations near the surface," Atmospheric Environment, vol. 51, pp. 56-66, 2012.

[11] D. Anfossi, S. Alessandrini, S. Trini Castelli, E. Ferrero, D. Oettl, and G. Degrazia, "Tracer dispersion simulation in low wind speed conditions with a new 2D Langevin equation system," Atmospheric Environment, vol. 40, no. 37, pp. 7234-7245, 2006.

[12] F. N. Frenkiel, "Turbulent diffusion: mean concentration distribution in a flow field of homogeneous turbulence," Advances in Applied Mechanics, vol. 3, pp. 61-107, 1953.

[13] R. J. Murgatroyd, "Estimations from geostrophic trajectories of horizontal diffusion in the mid-latitude troposphere and lower stratosphere," Journal of the Royal Meteorological Society, vol. 95, pp. 40-62, 1969.

[14] K. Manomaiphiboon and A. G. Russell, "Evaluation of some proposed forms of Lagrangian velocity correlation coefficient," International Journal of Heat and Fluid Flow, vol. 24, no. 5, pp. 709-712, 2003.

[15] G. A. Degrazia, A. Goulart, J. Costa Carvalho, C. R. P. Szinvelski, L. Buligon, and A. Ucker Timm, "Turbulence dissipation rate derivation for meandering occurrences in a stable planetary boundary layer," Atmospheric Chemistry and Physics, vol. 8, no. 6, pp. 1713-1721, 2008.

[16] D. J. Thomson, "Criteria for the selection of stochastic models of particle trajectories in turbulent flows," Journal of Fluid Mechanics, vol. 180, pp. 529-556, 1987. 
[17] E. Ferrero and D. Anfossi, "Comparison of PDFs, closure schemes and turbulence parameterizations in Lagrangian stochastic models," International Journal of Environment and Pollution, vol. 9, no. 4, pp. 384-410, 1998.

[18] C. W. Gardiner, Handbook of Stochastic Methods, Springer, Berlin, Germany, 1997.

[19] Z. Sorbjan, Structure of the Atmospheric Boundary Layer, Prentice-Hall, Englewood Cliffs, NJ, USA, 1989.

[20] H. F. Campos Velho, R. R. Rosa, F. M. Ramos et al., "Multifractal model for eddy diffusivity and counter-gradient term in atmospheric turbulence," Physica A, vol. 295, no. 1-2, pp. 219-223, 2001.

[21] C. Tsallis, "Possible generalization of Boltzmann-Gibbs statistics," Journal of Statistical Physics, vol. 52, no. 1-2, pp. 479-487, 1988.

[22] J. F. Sagendorf and C. R. Dickson, "Diffusion under low wind speed, inversion conditions," NOAA Technical Memorandum ERL ARL-52, 1974.

[23] P. Zannetti, Air Pollution Modeling. Teories, Computational Methods and Available Software, Kluwer Academic Publishers, 1990.

[24] S. S. Zilitinkevich, "On the determination of the height of the Ekman boundary layer," Boundary-Layer Meteorology, vol. 3, no. 2, pp. 141-145, 1972.

[25] H. C. Rodean, Stochastic Lagrangian Models of Turbulence Diffusion, American Meteorological Society, Boston, Mass, USA, 1996.

[26] S. R. Hanna, "Confidence limits for air quality model evaluations, as estimated by bootstrap and jackknife resampling methods," Atmospheric Environment, vol. 23, no. 6, pp. 1385-1398, 1989. 

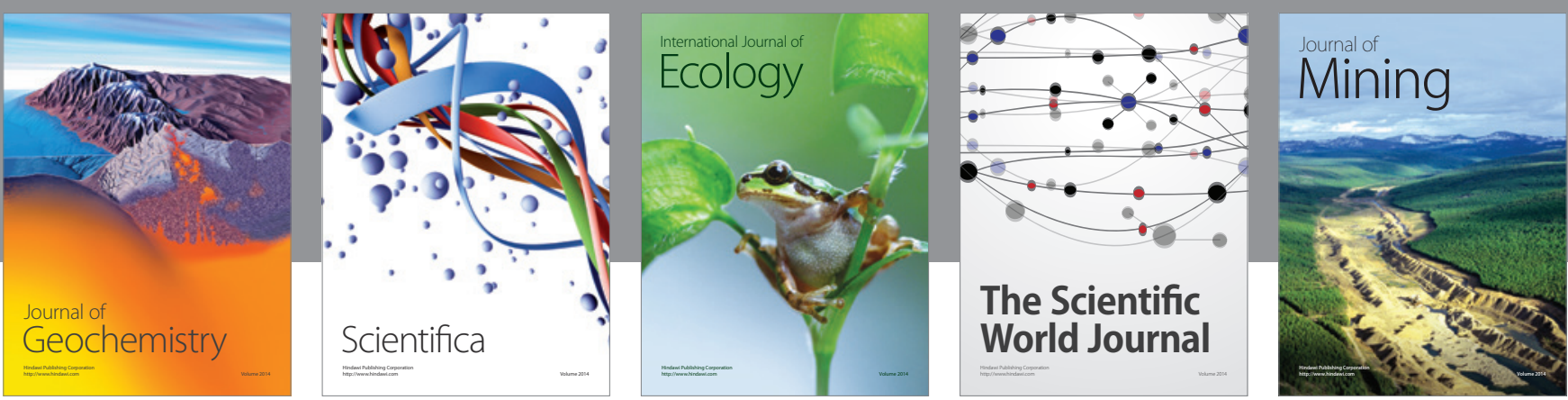

The Scientific World Journal
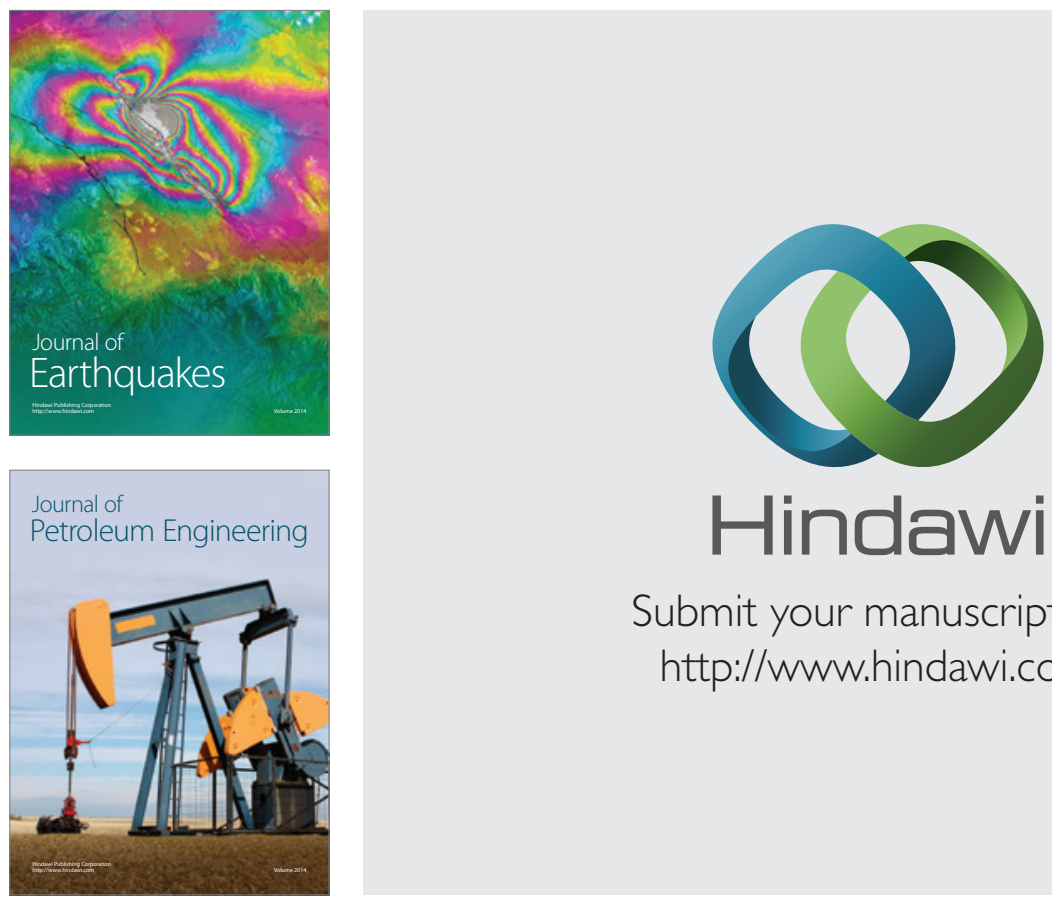

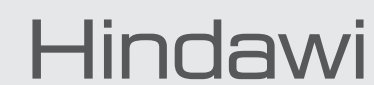

Submit your manuscripts at

http://www.hindawi.com
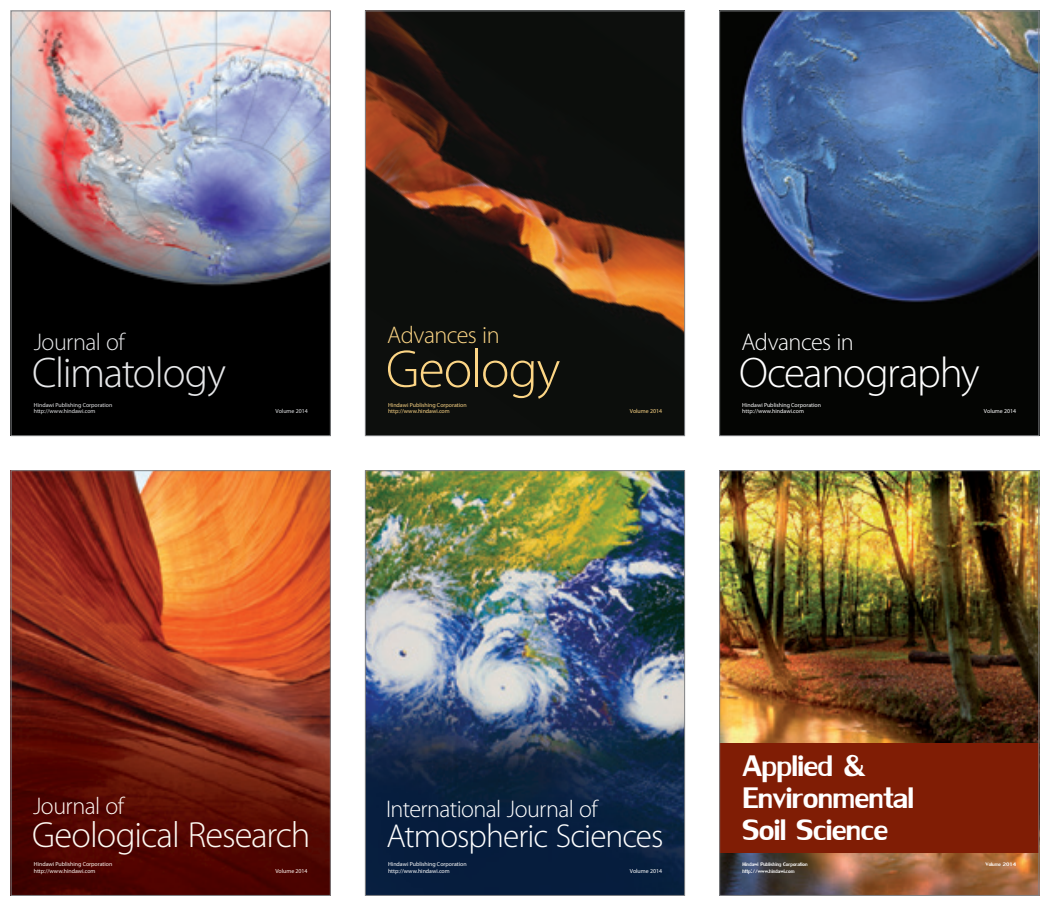
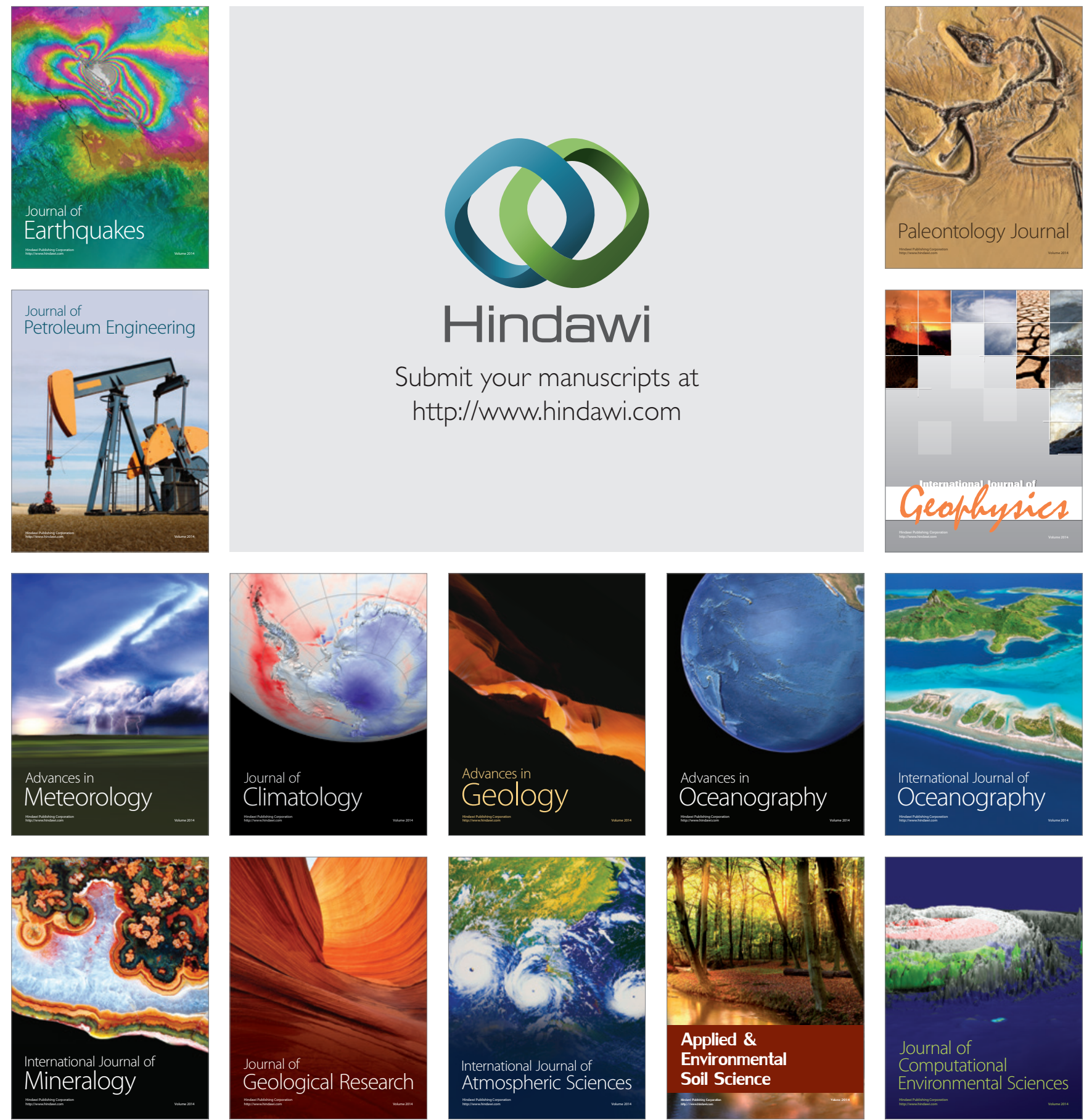